expression is important for the presentation of antigen in the induction of a cellular immune response. Because of the presence of a blood-brain barrier, circulating macrophages are unlikely to be involved in the initial presentation of antigen within the central nervous system.

Because the expression of class II molecules can be induced on the endothelial cells by contact with activated $\mathrm{T}$ lymphocytes, it is probable that this results in the release of lymphokines that break down the blood-brain barrier and hence leads to the invasion of blood-borne cells into the brain parenchyma.
As sophisticated techniques are now available both for isolating microvessels from the brain tissue and for culturing cerebral endothelial cells ${ }^{22}$, it seems likely that new therapeutic means will soon be developed to influence cerebral endothelial functions. Examples of such methods are to improve the transendothelial transport of particular substances and to reduce albumin extravasation in vasogenic brain oedema.

F. Joo is the Leader of the Laboratory of Molecular Neurobiology, Institute of Biophysics, Biological Research Center, 6701-Szeged Hungary.

\title{
Growth factors
}

\section{From the laboratory to the clinic}

\section{from Mike Dexter}

HORMONE therapy is used for a wide range of disorders and the results of treatment are often dramatic. The work primarily involves the classical 'glandular' hormones that are released as circulating messenger systems because fairly large amounts of material are readily available and their target cells and tissues are known. But many other important messenger systems modulate the behaviour and function of cells. Cytokines, for example, are soluble cell products that can modify the growth or behaviour of the same or unrelated cells. Thus the biological activities of lymphokines and monokines produced by lymphocytes and monocytes, respectively, have been elegantly elucidated in vitro. The problem is that these soluble mediators are often only produced locally and in vanishingly small amounts, making studies of their role in vitro and of their clinical usefulness formidable. But with the molecular cloning and expression in suitable vector systems of many of these molecules, sufficiently large amounts of material are now available to perform preclinical and clinical studies and this potential was highlighted at a recent meeting*

It is all very well being able to manipulate cells in vitro, but clinical application hinges on whether or not these agents can exert effects in vivo and if the effects observed can be predicted from the in vitro analyses. Under discussion were growth factors regulating $B$ and $T$ lymphocytes and tumour necrosis factor. Several general points should be noted. First, recombinant molecules essentially have the same biological activities and are just as effective as the native molecules irrespective of where, how or by whom they were cloned. Second, these cytokines do elicit responses in vivo - although sometimes with surprising consequences.

"International Workshon on (linical Application of I ympho kines and Cytokines. Erlangen. FRG:5-8 March 1986.
Third, many of the cytokines under consideration will almost certainly prove useful in the clinic, either used alone or in combinations - the problem is to define the disease, the dose and the route of administration.

Murine growth factors, interleukin-3 and granulocyte-macrophage colony stimulating factor (GM-CSF), which are active on stem cells or myeloid progenitor cells in vitro, will elicit responses in vivo (D. Metcalf, Melbourne; my own work; H. Broxmeyer, Indianapolis). Interleukin-3 activates stem cell cycling and promotes an increase in stem cells and progenitor cells in the spleen whereas GM-CSF can mobilize and recruit activated granulocytes and macrophages. Having established their activity in vivo, the next stage is to determine whether these agents, alone or in combination with macrophage colony stimulating factors (M-CSF) (P. Ralph, San Francisco), have therapeutic potential in enhancing blood cell generation in, for example, preor post-chemotherapy radiation, in the myeloid hypoplasias or in neutrophil deficiency syndromes. Furthermore, as macrophages and granulocytes play a major part in combating infestations with helminths, protozoa and numerous other colonizing organisms, the ability to activate the mobilize these cells with GM-GSF and $\mathrm{M}-\mathrm{CSF}$ has many profound implications. Many such studies are already underway using animals as models and it must be only a matter of time before the first clinical trials are performed. In this respect, recombinant human erythropoietin, a factor that enhances red blood cell production and works in vivo, seems to be a suitable starting material ( $\mathrm{J}$. Egrie, Thousand Oaks, United States) expecially for those patients with anaemia caused by kidney damage.

A recurring problem with many of these cytokines is their rapid clearance from the plasma coupled with the fact that cells must often be continuously exposed to the agents to elicit a response. But the pharmacokinetics are being studies and continuous infusion regimes are available to maintain a constant plasma level. This could be particulary important in the management of malignant disease. Interferon is very efficient for the treatment of hairy cell leukaemia (J. Quesada, Houston) and another cytokine (granulocyte-colony stimulating factor, or pluripoietin) has the remarkable property of being able to induce differentiation of myeloid leukaemic cells while at the same time amplifying the number of primitive normal progenitor cells in vitro (M. Moore, New York; E. Platzer, Erlangen). The potential use is obvious and the way is clear to undertake studies on the use of this regulator for 'leukaemia ablative therapy', in vitro, before autologous marrow transplantation (see Chang, J. et al. Lancet i, 294; 1986).

What about the interferons and other lymphokines? Clinical trials are underway to investigate the therapeutic potential of interferons in various malignant and nonmalignant diseases. With the exception of hairy cell leukaemia, the resuits are somewhat disappointing $(\mathrm{H}$. Oettgen, New York; P. Voldering, San Francisco; Robinson, Denver). But the lymphokines represent an interacting network of messenger signals capable of modulating the responses of one to the other. Interleukin-1, for example, stimulates myeloid progenitor cell development indirectly by stimulating the production of GM-CSF by macrophages (A. Billiau, Leaven). So part of the effects of treatment with interleukin-1 in vivo may actually mimic the effects of treatment with GM-CSF. Similarly, interleukin-2 receptors normally associated with $\mathrm{T}$ lympocytes are found on many other cell types that also can respond to interleukin-2. Also, tumour necrosis factor, which synergizes with $\gamma$-interferon, stimulates interleukin1 production and can activate the phagocytic activity of neutrophils (M. Palladino, San Francisco). A whole series of events, probably involving the generation of other cytokine messenger systems, obviously can be elicited following administration of a single cytokine. The problem is now to define some of these networks and to determine how the cytokines can be used most efficiently to promote the desired effect. This will not preclude further clinical studies provided that the situation is justified - but it will not be surprising if some of the effects seen are not quite as predicted from the assay systems in vitro.

Mike Dexter is Professor of Experimental Haematology at the Paterson Laboratories. Christie Hospital \& Holt Radium Institute, Manchester M20 9BX, UK. 